\title{
Study of Orbital Angular Momentum Mode Crosstalk Induced by Propagation Through Water
}

\author{
Shaun Viola ${ }^{\mathrm{a}}$, Manousos Valyrakis ${ }^{\mathrm{a}}$, Anthony E. Kelly ${ }^{\mathrm{a}}$, and Martin P.J. Lavery ${ }^{\mathrm{a}}$

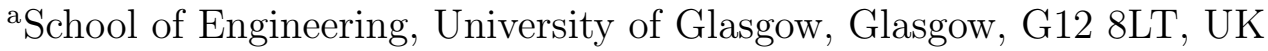

\begin{abstract}
The implementation of spatial multiplexing has become an area of great interest for free-space communication links, particularly for its use in last-mile links within larger optical networks. Light carrying orbital angular momentum (OAM) has emerged as a potential candidate that could be utilised for multiplexing independent channels. We will present measured inter-channel crosstalk for a set of 11-OAM modes propagating through $3 \mathrm{~m}$ of slowly flowing water, similar to that found in oceanic flow conditions.
\end{abstract}

Keywords: Orbital Angular Momentum, Visible Light Communications, Underwater Communications

\section{INTRODUCTION}

The drive for high-bandwidth, secure communications links has lead to space-division-multiplexing (SDM) becoming a burgeoning area of study. SDM techniques implemented in systems employing both optical and radiofrequency carriers have recently seen interest from the community for use within point-to-point communication links, particularly in long distance fibre links. ${ }^{1,2}$ Orbital angular momentum (OAM) multiplexing has emerged as a particular form of SDM that could be a potential candidate utilization for the multiplexing independent spatial channels in free-space optical, ${ }^{3-5}$ optical fibre ${ }^{6,7}$ and radio frequency ${ }^{8}$ systems. Such spatial modes with transverse a amplitude profile of $A(x, y) \exp (i \ell \phi)$, and carry angular momentum of $\ell \hbar$ per photon. ${ }^{9}$ The integer $\ell$ is unbounded giving a potentially unbounded number of independent multiplexed channels. ${ }^{3,10}$

In recent years the utilisation of visible light communications (VLC) has become an emerging technology with the potential to replace the currently widely implemented ultrasound underwater communication technology. ${ }^{11,12}$ Such VLC schemes could potentially be enhanced through the addition of OAM multiplexing. ${ }^{13,14}$ To assess the feasibility of such OAM multiplexing in an underwater communications channel it is important to analyse the degree of observed channel degradation induced by flowing water in real Oceanic conditions.

In this paper we present a study of measured inter-channel crosstalk for a set of OAM modes propagating through $3 \mathrm{~m}$ of slowly flowing water, similar to that found in Oceanic conditions.

\section{TURBULENCE EFFECTS ON BEAMS CARRYING OAM}

Free-space communications links encounter issues with atmospheric turbulence. This turbulence has been studied at great length by the astronomy community to correct the resulting aberrations introduced to an image. ${ }^{15}$ The natural randomly time dependent variations in temperature and pressure change the density of the atmosphere. This change in density results in a spatial dependent change of the refractive index; leading to phase distortions across a propagating optical beam. ${ }^{16}$ In specific cases, this turbulence can be simulated by a single phase screen and is commonly referred to as thin phase turbulence. ${ }^{17}$

The drive for the use of OAM as a method for multiplexing has spurred investigations into the affect such atmospheric turbulence on propagating beams carrying OAM. One can model atmospheric turbulence by considering as normal random variables, where the ensemble average can be written as $\left\langle\left[\phi\left(\mathbf{r}_{\mathbf{1}}\right)-\phi\left(\mathbf{r}_{\mathbf{2}}\right)\right]^{2}\right\rangle$ and is

Further author information: (Send correspondence to M.P.J.L.)

M.P.J.L.: E-mail: martin.lavery@glasgow.ac.uk, Telephone: +44 1413304799

Advanced Free-Space Optical Communication Techniques and Applications II, edited by Leslie Laycock, Henry J. White, Proc. of SPIE Vol. 9991, 999103

(C) 2016 SPIE $\cdot$ CCC code: $0277-786 X / 16 / \$ 18 \cdot$ doi: $10.1117 / 12.2245487$

Proc. of SPIE Vol. $9991999103-1$ 

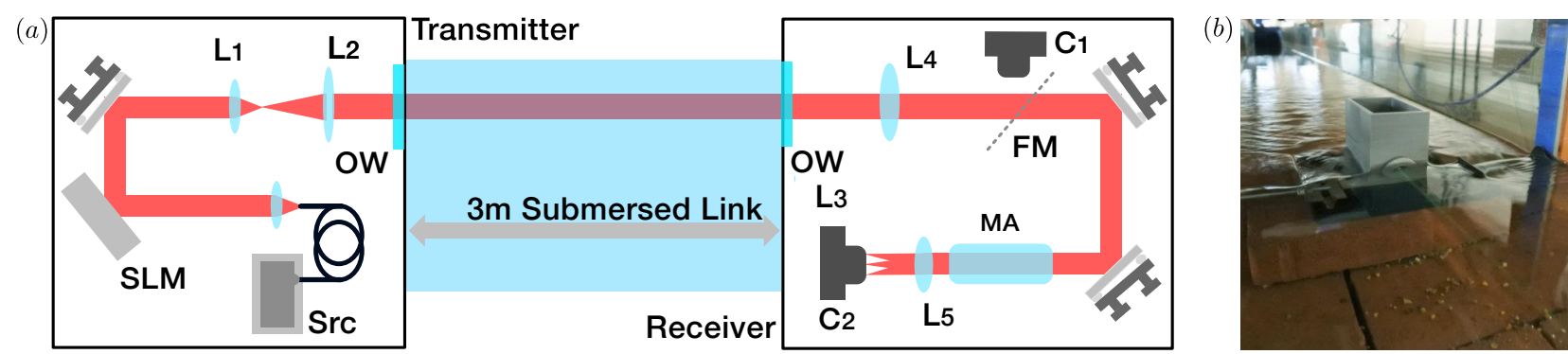

Figure 1. (a) A coherent laser source (Src) with a wavelength of $535 \mathrm{~nm}$ is coupled into a single mode fiber, and the output of the fiber collimated by a $25 \mathrm{~mm}$ lens. The collimated laser light is directed onto the surface of a spatial light modulator (SLM), which has an $\ell$-forked digital hologram encoded on it's optical surface to modulate the phase to the desired OAM mode. As the digital hologram is a diffraction grating, the first-order diffracted beam is spatially filtered at the focal plane of $L_{1}$. A second lens, $L_{2}$, of the same focal length is used to collimate the beam before propagation over the submersed link. To prevent modal degradation resulting from surface boundary effects, a Gorilla-glass optical window (OW) is used within a submersed 3D-printed water-proof enclosure, shown in (b). A field lens, $L_{4}$, for focal length $500 \mathrm{~mm}$ is used to mitigate divergence accumulated over the submersed link. A flip mirror (FM) is utilised to allow for images of the received mode to be collected by $C_{1}$. A combination of a mode analyser (MA) and a lens, $L_{5}$, are used to transform the received OAM modes in discreet positions on a fast camera, $C_{2}$.

known as the phase structure function. ${ }^{18}$ Where $\phi\left(\mathbf{r}_{\mathbf{1}}\right)$ and $\phi\left(\mathbf{r}_{\mathbf{2}}\right)$ are two randomly generated phase fluctuations. From Kolmogorov statistics it can be shown that this ensemble average must meet the requirement that

$$
\left\langle\left[\phi\left(\mathbf{r}_{1}\right)-\phi\left(\mathbf{r}_{2}\right)\right]^{2}\right\rangle=6.88\left|\frac{\mathbf{r}_{1}-\mathbf{r}_{2}}{r_{0}}\right|^{5 / 3}
$$

The value $r_{0}$ is the Fried parameter, and is a measure of the traverse distance scale over which the refractive

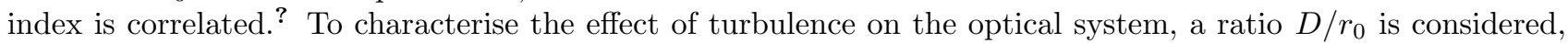
where $D$ is the aperture of the system. This ratio sets two limiting cases, first when $D / r_{0}<1$ the resolution of the system is limited by it's aperture, and second case of $D / r_{0}>1$, the atmosphere limits the systems ability to resolve an object. ${ }^{17}$

By considering the Fried parameter the spread in the OAM spectrum can be predicted by resulting from thin phase turbulence. ${ }^{19}$ Considering a single OAM mods, $\psi_{\ell}$, transmitted through an ensemble average of many turbulent phase screens, the power, $s_{\Delta}$, in the a particular mode, $\psi_{\ell+\Delta}$, is given by

$$
s_{\Delta}=\frac{1}{\pi} \int_{0}^{1} \rho \mathrm{d} \rho \int_{0}^{2 \pi} \mathrm{d} \theta e^{-3.44\left[\left(\frac{D}{r_{0}}\right)\left(\rho \sin \frac{\theta}{2}\right)\right]^{5 / 3}} \cos \Delta \theta,
$$

where $\Delta$ is a integer step in mode index of $\ell=1$, and $\rho=2 r / D .^{18}$ Subsequently experimental confirmation of this theory has been achieved. ${ }^{20}$ The development of mitigation of the effects on beams carrying OAM resulting from atmospheric turbulence has become an area of study. ${ }^{21,22}$

\section{EXPERIMENTAL DESIGN AND SYSTEM CHARATERISATION}

Our system comprises transmitter and receiver apertures placed $3 \mathrm{~m}$ apart, within a larger (approximately $15 \mathrm{~m}$ long, and $0.3 \mathrm{~m}$ wide) water tank, Fig. 1 . This tank has an adjustable flow regulator allowing control over the flow rate of the water with in the tank. We wish to study the modal degradation that occurs from transmission of an optical beam carrying OAM through flowing water similar in speed to that of the golf stream, with an average flow rate of $1.3 \mathrm{~ms}^{-1}$.

To prepare the required optical modes a spatially filtered $532 \mathrm{~nm}$ laser source illuminates the surface of a spatial light modulator. An $\ell$-forked hologram is displayed on the optical surface of the SLM, generating the required OAM mode. ${ }^{23}$ The optical mode is relayed using a periscopic mirror arrangement into a 3D-Printed water-proof enclosure, Fig. 1 (b). Transmission through an air-to-water boundary results in considerable optical 

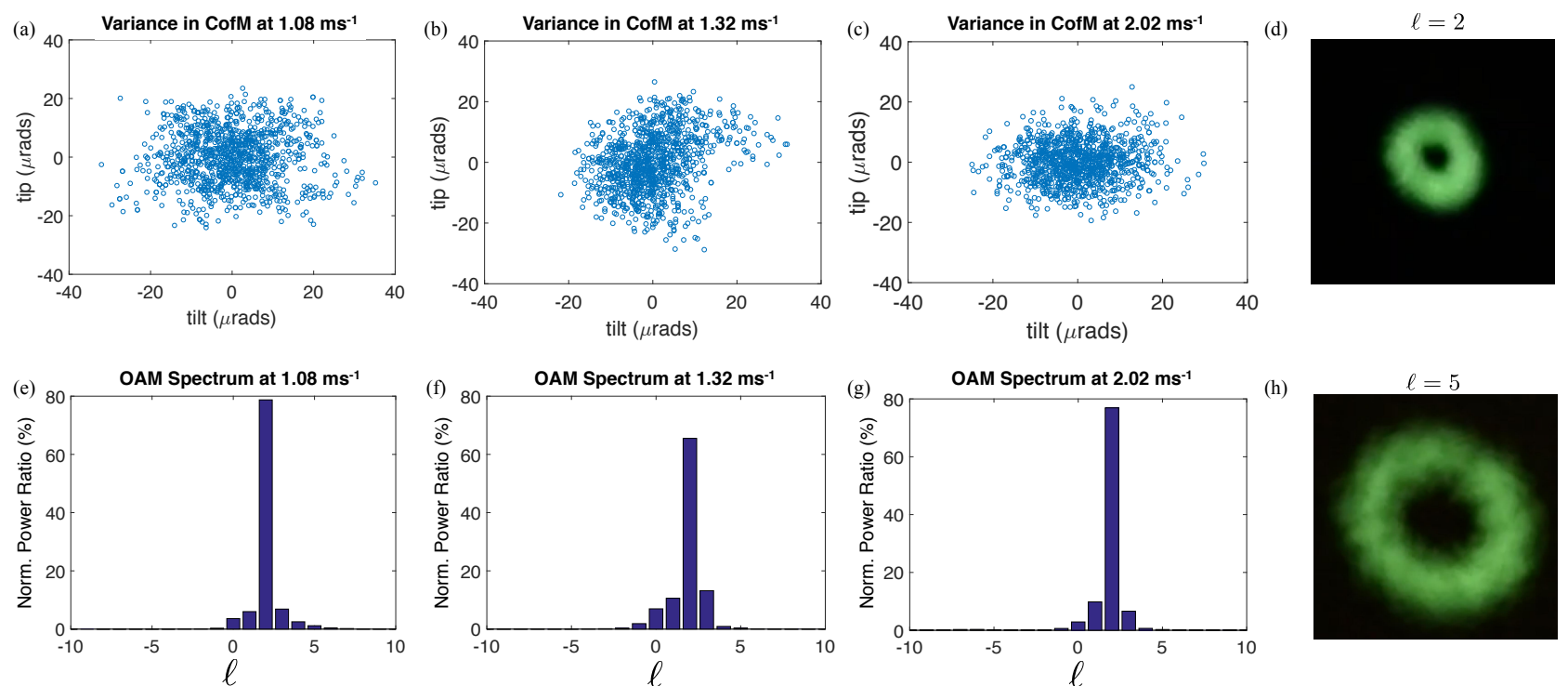

Figure 2. (a-c) Tip-tilt aberration are a leading form of modal degradation experienced by free-space optical systems, submersed links are similarly affected by this form of turbulence. $C_{1}$ records the intensity profile of the received optical beam at intervals of $16 \mu \mathrm{s}$, allowing for the degree of tip-tilt aberration to be determined. A centroid measurement was calculated from each recorded frame, and plotted as a scatter plot. Images of the received optical modes for optical modes (d) $\ell=2$ and (h) $\ell=5$. (e-g) For each flow rate, the modal crosstalk can be determined by the intensity profile on camera, $C_{2}$. The measured intensity is averaged over 1200 frames for each speed respectively.

(a) Channel Power Matrix at $1.08 \mathrm{~ms}^{-1}$

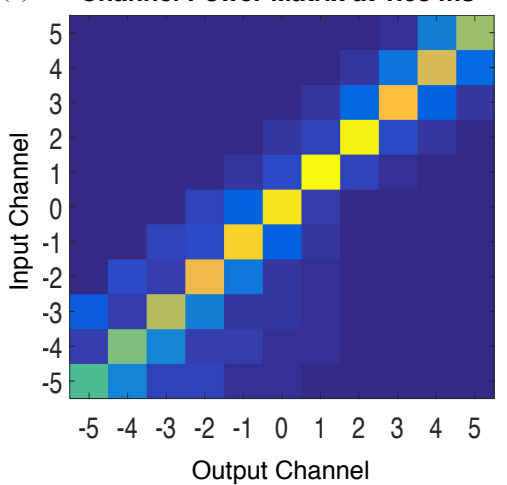

(b) Channel Power Matrix at $1.32 \mathrm{~ms}^{-1}$

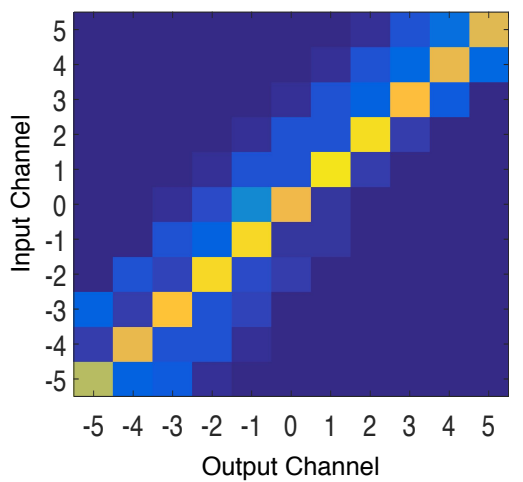

(c) Channel Power Matrix at $2.02 \mathrm{~ms}^{-1}$

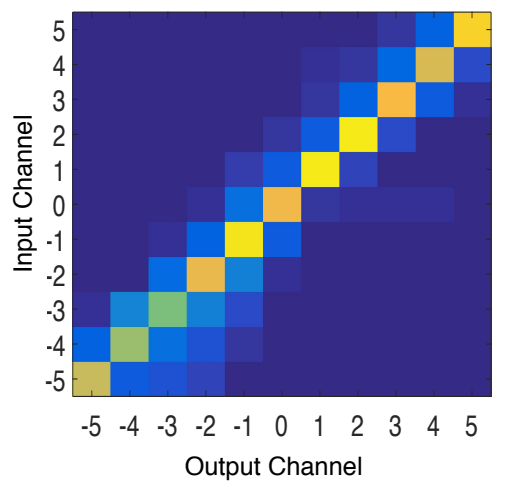

Figure 3. (a-c) A crosstalk matrix can be generated to visualise the expected crosstalk for each of the 11 OAM channels for flow rates $1.08 \mathrm{~ms}^{-1}, 1.32 \mathrm{~ms}^{-1}$ and $2.02 \mathrm{~ms}^{-1}$ respectively. The measured crosstalk is the averaged modal crosstalk over a $10 \mathrm{~s}$ measurement window. Hence, crosstalk arising from tip-tilt aberrations and static system aberrations both contribute to the crosstalk measured. 

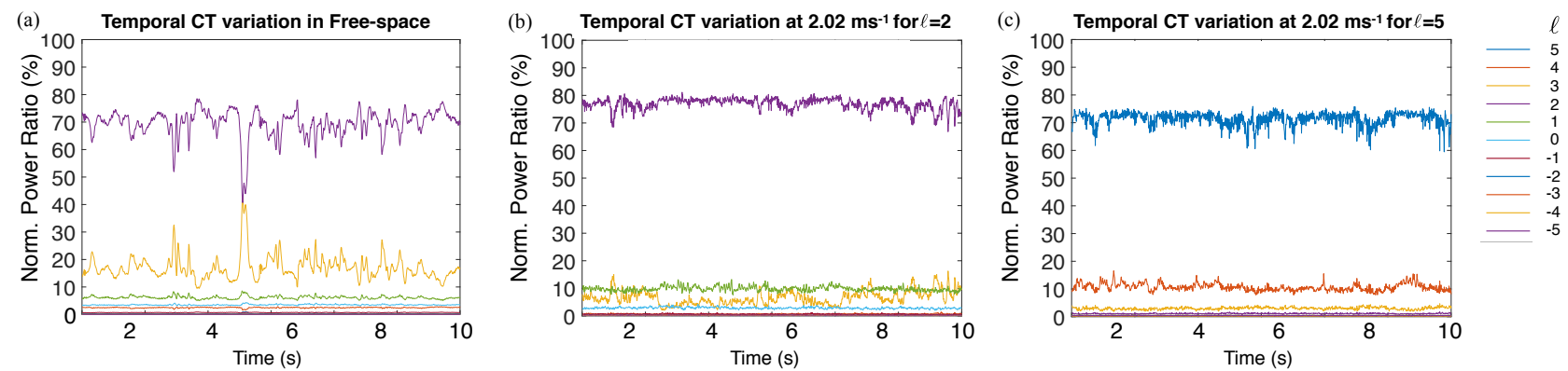

Figure 4. System stability is an important consideration. To investigate the stability of the submersed link we consider the channel crosstalk measured at each individual frame. Each frame has $16 \mu \mathrm{s}$ exposure time. It can be seen that the free-space link (a) has greater frame-by-frame variation in modal crosstalk as compared to a link that has water flowing at $2.02 \mathrm{~ms}^{-} 1$ (b). We further consider an OAM mode with $\ell=5$, showing the stability is broadly similar for the higher order modes.

aberrations due to turbidity on the water surface, hence our optical modes are passed through a submersed optical window reducing the effects from the fluctuating water surface.

Within any point-to-point to communications link, tip-tilt aberration is one of the largest concerns. Within oceanic conditions, the flow of the water potentially places added stress on the optical system and could perturb the optical beam resulting in tip-tilt aberrations. We first characterize the degree of tip-tilt aberration within the link by tracking the Centre of Mass (CofM), at $16 \mu$ s intervals, of collected beam over a measurement time of 10-seconds for flow rates of $1.08 \mathrm{~ms}^{-1}, 1.38 \mathrm{~ms}^{-1}$ and $2.02 \mathrm{~ms}^{-1}$ respectively, Fig. 2 (a-c). The variation in CofM indicates a misalignment between the beam axis, the centre of the propagating mode, and the measurement axis of the system, where such misalignment will result in channel crosstalk. ${ }^{25}$

The OAM spectrum after propagation over the submersed link was measured with the use of an OAM mode sorter. The mode sorter uses two refractive optical elements that transform OAM states into transverse momentum state. ${ }^{?, 24}$ These optical elements transform both the phase and intensity of the beam in the form $\exp (i \ell \theta)$, to give a complex amplitude at the output plane of the form $\exp (i \ell x / a)$, where $a$ is a scaling parameter. A lens then can separate these resulting transverse momentum states into specific lateral positions. A camera is placed at the focal plane of this lens, where eleven, adjacent, equally sized regions were selected, each corresponding to a specific mode index. The total power over all the pixel in each region was summed to give the power in that mode, allowing for the efficient measurement of the constituent OAM states simultaneously. ${ }^{25}$

A mode range of $\ell=-5$ to $\ell=+5$ was chosen, and the power measured in each detection regions was averaged over a 10 second measurement window. Independent of turbulence, our mode sorter has an residual degree crosstalk between channels due to the diffraction limit, and issues arising from skew angle of the rays.?,26 The channel crosstalk for each of the 11-modes was measured for the three test flow rates to determine the experimental crosstalk, Fig. 3 (a-c). It can be seen that the measured crosstalk within the optical system does not vary drastically when measured at the different flow rates investigated. This lack of variation indicated that it is inherent system aberrations and in-stabilities in the optical setup that are leading to the observed channel crosstalk.

\section{SYSTEM STABILITY AND TRANSMISSION POWER LOSS}

To determine the contribution of mechanical stability on the measured average crosstalk measured in the optical system, we investigate the crosstalk that occurs during every frame measured at camera $\mathrm{C}_{2}$. The fluctuation in modal crosstalk with respect to time can give an indication of the relative mechanical movement between the transmitter and the receiver. We consider this for free-space, where no water is flowing through the channel and for the case of water flowing at $2.02 \mathrm{~ms}^{-1}$, Fig. 4 (a) and (b) respectively. It can be seen that there is less fluctuations in the case where water is flowing in the channel. We believe the weight of the water flowing in the channel, mechanically stabilises the system reducing the degree of modal crosstalk observed. This indicates that under the water conditions similar in form to those found in coastal waters, the key task is the mitigation of tip-tilt aberrations arising from relative mechanical movement between the transmitter and receiver. 

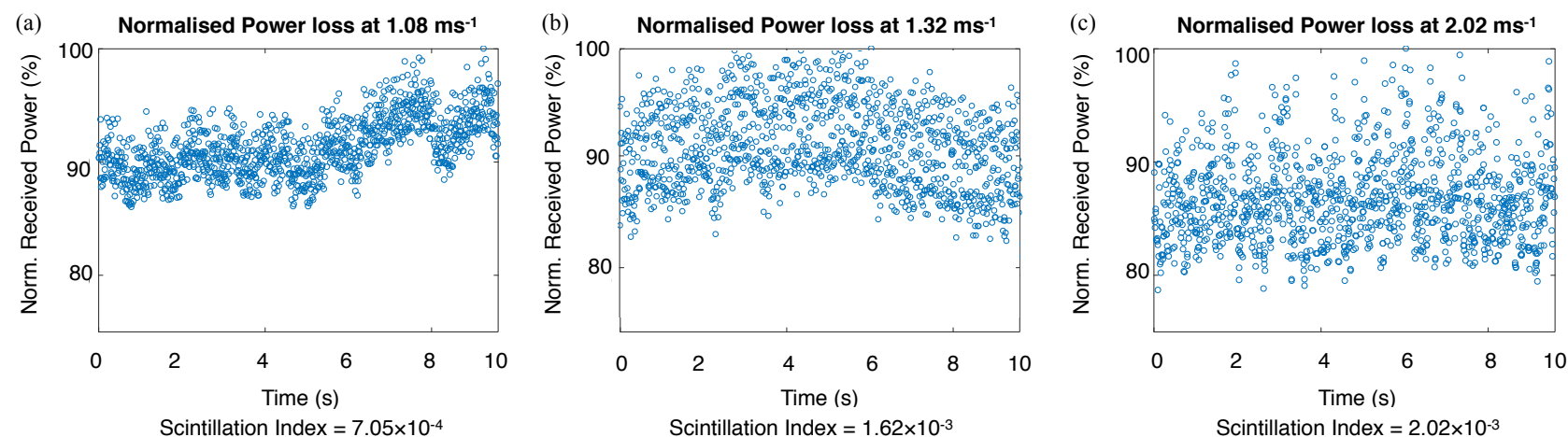

Figure 5. Optical scattering from particulates are a concern with a submersed optical link. These particles move with the fluid flow and can result in a variance in measured crosstalk and power loss. The normalised power is considered frame-by-frame for flow speeds $1.08 \mathrm{~ms}^{-1}, 1.32 \mathrm{~ms}^{-1}$ and $2.02 \mathrm{~ms}^{-1}$.

A further concern in the implementation of a point-to-point communications link is the loss of optical power as the light propagates over the submersed channel. We can consider the relative power fluctuation over our $10 \mathrm{~s}$ measurement window. This fluctuation can give an indication of the affect of natural buoyant particulates in the water channel. Small suspended particles scatter the light reducing the overall power in the system, along with a frame-by-frame variance resulting from their movement with water flows. The scintillation index is a calculated value that allows the evaluation of the temporal power fluctuation. We consider the power loss and scintillation index for each of the tested flow rates, shown in Fig. 5 (a-c) respectively.

\section{CONCLUSIONS}

In conclusion we have experimentally measured the crosstalk between OAM modes propagating through low discharge turbulent flows. Our initial results indicate that tip-tilt aberration is a considerable contributing factor to the expected inter-channel crosstalk. In coastal conditions, we expect there to be little modal degradation arising from phase aberrations. However, we expect mechanical stability of the optical system to be a central source of modal crosstalk.

\section{ACKNOWLEDGMENTS}

M.P.J.L. acknowledges support from the Royal Academy of Engineering. M.P.J.L. and S.V. acknowledge support from the EPSRC.

\section{REFERENCES}

[1] Richardson, D. J., Fini, J. M., and Nelson, L. E., "Space-division multiplexing in optical fibres," Nature Photonics 7, 354-362 (May 2013).

[2] Lavery, M. P. J., Huang, H., Ren, Y., Xie, G., and Willner, A. E., "Demonstration of a 280Gbit/s freespace space-division-multiplexing communications link utilizing plane-wave spatial multiplexing," Optics Letters 41, 851-854 (Mar. 2016).

[3] Gibson, G., Courtial, J., Padgett, M. J., Vasnetsov, M. V., Pas'ko, V. A., Barnett, S. M., and FrankeArnold, S., "Free-space information transfer using light beams carrying orbital angular momentum," Optics Express 12(22), 5448-5456 (2004).

[4] Wang, J., Yang, J.-Y., Fazal, I. M., Ahmed, N., Yan, Y., Huang, H., Ren, Y., Yue, Y., Dolinar, S., Tur, M., and Willner, A. E., "Terabit free-space data transmission employing orbital angular momentum multiplexing," Nature Photonics 6, 488-496 (June 2012).

[5] Huang, H., Xie, G., Yan, Y., Ahmed, N., Ren, Y., Yue, Y., Rogawski, D., Willner, M. J., Erkmen, B. I., Birnbaum, K. M., Dolinar, S. J., Lavery, M. P. J., Padgett, M. J., Tur, M., and Willner, A. E., "100Tbit/s free-space data link enabled by three-dimensional multiplexing of orbital angular momentum, polarization, and wavelength," Optics Letters 39, 197-200 (Jan. 2014). 
[6] Bozinovic, N., Yue, Y., Ren, Y., Tur, M., Kristensen, P., Huang, H., Willner, A. E., and Ramachandran, S., "Terabit-Scale Orbital Angular Momentum Mode Division Multiplexing in Fibers," Science 340, 1545-1548 (June 2013).

[7] Huang, H., Milione, G., Lavery, M. P. J., Xie, G., Ren, Y., Cao, Y., Ahmed, N., Nguyen, T. A., Nolan, D. A., Li, M.-J., Tur, M., Alfano, R. R., and Willner, A. E., "Mode division multiplexing using an orbital angular momentum mode sorter and MIMO-DSP over a graded-index few-mode optical fibre," Scientific Reports 5 (2015).

[8] Yan, Y., Xie, G., Lavery, M. P. J., Huang, H., Ahmed, N., Bao, C., Ren, Y., Cao, Y., Li, L., Zhao, Z., Molisch, A. F., Tur, M., Padgett, M. J., and Willner, A. E., "High-capacity millimetre-wave communications with orbital angular momentum multiplexing," Nature Communications 5, 4876 (Sept. 2014).

[9] Allen, L., Beijersbergen, M. W., Spreeuw, R. J. C., and Woerdman, J. P., "Orbital angular-momentum of light and the transformation of Laguerre-Gaussian laser modes," Physical Review A 45(11), 8185-8189 (1992).

[10] Yao, A. M. and Padgett, M. J., "Orbital angular momentum: origins, behavior and applications," Advances in Optics and Photonics 3(2), 161-204 (2011).

[11] Hanson, F. and Radic, S., "High bandwidth underwater optical communication," Applied Optics 47, 277-283 (Jan. 2008).

[12] Watson, S., Tan, M., Najda, S. P., Perlin, P., Leszczynski, M., Targowski, G., Grzanka, S., and Kelly, A. E., "Visible light communications using a directly modulated $422 \mathrm{~nm}$ GaN laser diode," Optics Letters 38, 3792-3794 (Oct. 2013).

[13] Ren, Y., Li, L., Zhao, Z., Xie, G., Wang, Z., Ahmed, N., Yan, Y., Willner, A., Cao, Y., Liu, C., Ashrafi, N., Ashrafi, S., Tur, M., and Willner, A., "4 Gbit/s Underwater Transmission Using OAM Multiplexing and Directly Modulated Green Laser," Conference on Lasers and Electro-Optics (2016), paper SW1F.4, SW1F.4 (June 2016).

[14] Ren, Y., Li, L., Wang, Z., Kamali, S. M., Arbabi, E., Arbabi, A., Zhao, Z., Xie, G., Cao, Y., Ahmed, N., Yan, Y., Liu, C., Willner, A. J., Ashrafi, S., Tur, M., Faraon, A., and Willner, A. E., "Orbital Angular Momentum-based Space Division Multiplexing for High-capacity Underwater Optical Communications," arXiv.org (Apr. 2016).

[15] Beckers, J. M., "ADAPTIVE OPTICS FOR ASTRONOMY: Principles. Performance and Applications," Annual review of astronomy and astrophysics 31, 13-62 (1993).

[16] Tatarski, V. I., "Wave Propagation in a Turbulent Mediumr," McGraw-Hill, New York (Apr. 1961).

[17] Fried, D. L., "Statistics of a Geometric Representation of Wavefront Distortion," JOSA 55, 1427-1431 (Nov. 1965).

[18] Tyler, G. A. and Boyd, R. W., "Influence of atmospheric turbulence on the propagation of quantum states of light carrying orbital angular momentum," Optics Letters 34, 142-144 (Jan. 2009).

[19] Paterson, C., "Atmospheric Turbulence and Orbital Angular Momentum of Single Photons for Optical Communication," Physical Review Letters 94, 153901 (2005).

[20] Rodenburg, B., Lavery, M. P. J., Malik, M., O’Sullivan, M. N., Mirhosseini, M., Robertson, D. J., Padgett, M. J., and Boyd, R. W., "Influence of atmospheric turbulence on states of light carrying orbital angular momentum," Optics Letters 37, 3736-3737 (May 2012).

[21] Ren, Y., Huang, H., Xie, G., Ahmed, N., Yan, Y., Erkmen, B. I., Chandrasekaran, N., Lavery, M. P. J., Steinhoff, N. K., Tur, M., Dolinar, S., Neifeld, M., Padgett, M. J., Boyd, R. W., Shapiro, J. H., and Willner, A. E., "Atmospheric turbulence effects on the performance of a free space optical link employing orbital angular momentum multiplexing," Optics Letters 38(20), 4062-4 (2013).

[22] Huang, H., Cao, Y., Xie, G., Ren, Y., Yan, Y., Bao, C., Ahmed, N., Neifeld, M. A., Dolinar, S. J., and Willner, A. E., "Crosstalk mitigation in a free-space orbital angular momentum multiplexed communication link using 44 MIMO equalization," Optics Letters 39(15), 4360-4 (2014).

[23] Heckenberg, N. R., McDuff, R., Smith, C. P., and White, A. G., "Generation of optical-phase singularities by computer-generated holograms," Optics Letters 17(3), 221-223 (1992). 
[24] Lavery, M. P. J., Robertson, D. J., Berkhout, G. C. G., Love, G. D., Padgett, M. J., and Courtial, J., "Refractive elements for the measurement of the orbital angular momentum of a single photon," Optics Express 20(3), 2110-2115 (2012).

[25] Lavery, M. P. J., Berkhout, G. C. G., Courtial, J., and Padgett, M. J., "Measurement of the light orbital angular momentum spectrum using an optical geometric transformation," Journal of Optics 13, 064006 (June 2011).

[26] Padgett, M. J., "The Poynting vector in Laguerre-Gaussian laser modes," Optics Communications 121, 36-40 (Nov. 1995). 